Revue d'histoire de l'Amérique française

Q REVUE D'HISTOIRE DE L'AMÉRIQUE FRANÇAISE

\title{
Un hebdomadaire libéral : L'Écho du Manitoba (1898-1905)
}

\section{Bernard Penisson}

Volume 28, numéro 3, décembre 1974

URI : https://id.erudit.org/iderudit/303368ar

DOI : https://doi.org/10.7202/303368ar

Aller au sommaire du numéro

Éditeur(s)

Institut d'histoire de l'Amérique française

ISSN

0035-2357 (imprimé)

1492-1383 (numérique)

Découvrir la revue

Citer cette note

Penisson, B. (1974). Un hebdomadaire libéral : L’Écho du Manitoba (1898-1905).

Revue d'histoire de l'Amérique française, 28(3), 367-384.

https://doi.org/10.7202/303368ar d'utilisation que vous pouvez consulter en ligne.

https://apropos.erudit.org/fr/usagers/politique-dutilisation/ 
NOTES DE RECHERCHE

I

UN HEBDOMADAIRE LIBÉRAL :

L'ÉCHO DU MANITOBA

$(1898-1905)^{*}$

Bernard Penisson

Agrégé de l'Université

Collège de Saint-Boniface

Manitoba

$\mathrm{Au}$ cours de recherches sur "La France devant l'opinion manitobaine", il nous a semblé que tout un secteur de la presse franco-manitobaine, la presse libérale, avait disparu presque totalement de la conscience collective et en grande partie des dépôts d'archives, et n'avait pas encore fait son apparition dans les travaux des historiens ${ }^{1}$. On ne la mentionnait qu'en passant, pour ironiser sur son insignifiance ou sur la personnalité douteuse de ses rédacteurs, confondus avec d'aimables bouffons sans expérience ou de dangereux adversaires de la cause catholique et française. Cette presse fut-elle vraiment de peu d'importance ? Pourquoi a-t-elle disparu des mémoires? La survivance des minorités francophones exige-t-elle le monopole d'un journal, la concurrence étant considérée moins comme un stimulant que comme un facteur de division des forces nationales? Même s'il est actuellement difficile de répondre à ces questions, il n'est peutêtre pas sans intérêt de les poser.

La presse canadienne-française naquit presque en même temps que la province, avec $L e$ Métis, puis Le Manitoba ${ }^{2}$. Ces

* Cette étude a été préparée à partir d'une communication présentée à la Société historique de Saint-Boniface, au cours du Colloque sur "L'idéologie et le rôle de la presse écrite au Manitoba français", organisé par Robert Painchaud le 22 novembre 1973.

1 A l'exception de la remarquable thèse de maîtrise, non publiée, de Roger E. Turenne, The Minority and the Ballot Box: A Study of the Voting Behaviour of the French Canadians of Manitoba, 1888-1967 (University of Manitoba, 1969), 184 p., qui utilise deux hebdomadaires libéraux: Le Courrier du Nord-Ouest et L'Echo du Manitoba.

2 Jean-Paul Aubry, o.m.i., "Presse francophone au Manitoba", Vie française 26 (janvier-février 1972): 115-136. Le Métis parut de 1871 à 1881 et Le Manitoba lui succéda de 1881 à 1925.

\section{[367]}

RHAF, vol. 28, no 3 (décembre 1974) 
journaux soutinrent rapidement les positions du groupe qui allait devenir le parti conservateur. En réaction contre le monopole du journal Le Manitoba, fondé par le député de Saint-Boniface, Alphonse Larivière ${ }^{3}$, les libéraux francophones tentèrent à plusieurs reprises de lancer des hebdomadaires. Leur durée fut plutôt brève. C'est ainsi que le député du comté de La Vérendrye, James Prendergast ${ }^{4}$, publia le 13 avril 1887, Le Trappeur, qui disparut très vite, après le deuxième numéro. Ce journal présente surtout l'intérêt de révéler que Le Manitoba ne convenait plus à quelques leaders de la minorité française; il est aussi le premier hebdomadaire libéral de langue française paru au Manitoba.

L'année suivante, l'ex-député de Sainte-Agathe, Ernest Cyr 5 , dirigea Le Courrier du Nord-Ouest (31 mai - 27 décembre 1888), qui se donnait pour mission d'organiser systématiquement l'aile francophone du parti libéral manitobain. Les libéraux dirigés par Thomas Greenway venaient de former le gouvernement du Manitoba (19 janvier 1888) et le premier ministre, conformément à la tradition de la province, avait choisi un Canadien d'ascendance française, James Prendergast, comme secrétaire provincial. "Il va sans dire, écrivait Le Courrier du Nord-Ouest, que notre journal est publié entièrement dans l'intérêt du gouvernement Greenway-Prendergast." Mais Saint-Boniface restait un bastion conservateur et, aux élections provinciales du 11 juillet 1888, Ernest Cyr mordait la poussière devant Roger Marion qui l'emportait par 19 voix de majorité. Peut-on conjecturer que c'est à la suite de cette défaite de son rédacteur et de "l'hostilité persistante et sourde dont le parti [libéral] [fut] l'objet dans

3 Alphonse-Alexandre-Clément Larivière (1842-1925), né à Montréal; arrivé au Manitoba en 1870; premier rédacteur du journal Le Manitoba; député provincial, 1878-1888; secrétaire provincial, 1881-1883; ministre de l'agriculture, 1883-1886; trésorier provincial, 1886-1887; député fédéral, 1889-1904; sénateur, 1911-1917; décédé à Saint-Boniface.

4 James-Emile-Pierre Prendergast (1858-1945); né à Québec; bachelier en droit, 1881; arrivé à Saint-Boniface en mars 1882; député provincial du comté de La Vérendrye, 1885-1888; du comté de Woodlands, 18881892; du comté de Saint-Boniface, 1892-1896; maire de Saint-Boniface, 1893-1896; juge à la cour du comté à partir de 1897; juge en chef du Manitoba, 1930-1944; décédé à Saint-Boniface.

5 Joseph-Ernest Cyr (1854-1929), né à Montréal; arrivé au Manitoba en mars 1882; député provincial de Sainte-Agathe, 1883-1886; maire de Saint-Boniface, 1885; défait aux élections fédérales de 1887 dans Provencher par Joseph Royal; défait aux élections provinciales de 1888 dans Saint-Boniface par Roger Marion; député fédéral de Provencher, 19041908; décédé à Saint-Boniface. 
certains quartiers" ", que Le Courrier $d u$ Nord-Ouest disparut le 27 décembre de la même année?

Malgré ces difficultés, Cyr et Prendergast fondèrent L'Ouest Canadien dont vingt-six numéros parurent du 14 février au 14 août 1889. Manifestement, les libéraux français jouaient de malchance, car c'est alors que furent rendus publics les projets de loi antifrançais et anticatholiques du cabinet libéral Greenway. Ces projets modifiaient profondément la constitution manitobaine de 1870, l'un, en enlevant au français son statut de langue officielle, l'autre, en réservant les subventions de l'Etat aux écoles neutres. Prendergast, alors député de Woodlands et toujours secrétaire provincial, donna sa démission à Greenway et saborda son journal en expliquant aux lecteurs:

Ce journal a été fondé pour appuyer le mouvement qui, en janvier 1888, ralliait nos destinées à celles du cabinet qui venait alors de se constituer.

On connaît trop, sans pouvoir en prédire encore toute la portée les événements malheureux qui bouleversent à cette heure notre monde politique. Ils rendent notre alliance avec le gouvernement désormais impossible, en lui enlevant toutes les garanties sur lesquelles elle reposait.

[...] Nous avons donc résolu de suspendre la publication de L'Ouest Canadien ${ }^{7}$.

Pendant plus de sept ans, ce fut pour les libéraux francomanitobains la traversée du désert. Mais, à la suite de la victoire de Wilfrid Laurier aux élections fédérales de juin 1896 et du règlement boiteux Laurier-Greenway de novembre suivant, les libéraux franco-manitobains refirent surface. La même année, ils constituèrent l'Association libérale française et, en 1898, ils fondèrent un nouveau journal, L'Echo de Manitoba.

\section{I - HISTORIQUE DE \\ L'ÉCHO DU MANITOBA}

Cet hebdomadaire a tenu une place importante sur la scène franco-manitobaine pendant près de huit ans, soit du 27 janvier 1898 au 20 juillet 1905. Fondé par le député libéral de Morris,

${ }^{6}$ L'Ouest Canadien (14 février 1889), 2. Le quartier en question est Saint-Boniface.

7 Ibid. (14 août 1889), 2. 
Alphonse Martin ${ }^{8}$, et par le commerçant Noé Chevrier ${ }^{9}$, propriétaire du "Magasin Bleu" à Winnipeg, le journal porta le nom de $L$ 'Echo "de" Manitoba jusqu'au 19 novembre 1903. Il arborait une devise qui fait sourire en tête d'un journal politique partisan: "Tout droit". Son premier directeur, Ernest Cyr, venait d'être élu, en 1896, premier président de l'Association libérale française du Manitoba. Très combatif, L'Echo servit le parti libéral comme instrument d'implantation grâce à son réseau de correspondants locaux présents dans quelques centres francophones de la province, comme Saint-Claude. Deux ans après la fondation du journal, en décembre 1899, les conservateurs menés par Hugh John Macdonald et Redmond Roblin remportèrent les élections provinciales, dans vingt-trois comtés contre quinze députés libéraux. ${ }^{10} L ' E c h o$ se trouvait désormais dans l'opposition sur le plan provincial et du côté du pouvoir sur le plan fédéral.

Tout comme ses prédécesseurs disparus, L'Echo voulait présenter à ses lecteurs l'aspect des problèmes que Le Manitoba passait sous silence, et combler ainsi une lacune importante de l'information provinciale de langue française. L'Echo compta un nombre usuel de six pages en 1898-1899, puis de huit pages à partir du 12 octobre 1899 jusqu'en novembre 1903, soit le double de son rival, Le Manitoba; il retomba dès lors à six pages jusqu'à sa disparition en juillet 1905. Par contre, le format de $L^{\prime} E c h o$ grandit continuellement et le nombre de colonnes par page passa de cinq à six, puis à sept. A partir du 1er août 1901, $L^{\prime} E c h o$ fit une place en première page aux reproductions photographiques, à la différence du journal Le Manitoba toujours privé d'illustrations.

L'Echo eut d'abord le même imprimeur que Le Manitoba, Antoine Gauvin, qui de la rue Main, à Winnipeg, transporta ses presses rue Provencher, à Saint-Boniface, après l'incendie de son atelier en janvier 1900. Puis, du 4 juillet 1901 au 13 novembre 1903, L'Echo fut imprimé à Ottawa par la Compagnie d'Imprimerie générale, qui éditait aussi le journal libéral Le Temps. Mais les lecteurs manitobains se plaignirent des délais de livraison occasionnés par la grande distance séparant l'imprimerie de la

8 Député provincial de Sainte-Anne, 1874-1879; de Morris, 1886-1901; battu en 1901 dans le comté de Woodlands par le premier ministre conservateur Roblin.

9 Noé Chevrier (1846-1911), né à Rigaud; arrivé au Manitoba vers 1873; sénateur, 1909-1911; décédé à Winnipeg.

10 Victoire confirmée en juillet 1903 par l'élection de 31 conservateurs. Les libéraux manitobains ne revinrent au pouvoir qu'en 1915. 
rédaction du journal. Le rédacteur, Henri d'Hellencourt, fit alors l'acquisition d'une linotype et imprima lui-même le journal à Winnipeg, du 26 novembre 1903 au 6 juillet 1905. D'Hellencourt fut sans doute embauché dès les débuts du journal, mais son nom n'apparaît pour la première fois que sur le numéro 47 , du 29 décembre 1898.

Comme il se devait, L'Echo fit une large publicité à l'Association libérale française et il connut ses heures de gloire lors de la tenue de la convention libérale franco-manitobaine à Winnipeg, le 23 juillet 1902, où près d'une centaine de délégués de 27 villes ou villages furent présents.

$L^{\prime} E c h o$ n'avait pas peu contribué en effet à donner une image prestigieuse, sinon à rendre le groupe libéral francomanitobain plus influent et cette convention lui donna l'occasion de faire le point. "Les libéraux français du Manitoba, écrivait d'Hellencourt, ont le droit d'être fiers des preuves de vitalité et d'intelligente initiative qu'ils viennent de donner en organisant la convention du 23 juillet ${ }^{11}$." Et, après un court historique des déboires du parti depuis 1888, déboires dont il rendait responsables les chefs conservateurs francophones qui avaient combattu les libéraux anglophones et rendu ainsi les lois de 1890 inévitables, il ajoutait:

Ce n'est en réalité qu'après 1896 , que les libéraux français adoptant la manière de voir du parti libéral au fédéral, se rallièrent et commencèrent à se compter. Depuis lors le succès a répondu à leur attente et aux dernières élections provinciales, les trois comtés français élurent des députés libéraux ${ }^{12}$.

Puis, d'Hellencourt affirmait que la minorité francophone devait prendre en mains ses propres intérêts et les défendre elle-même: les libéraux francophones essayant de faire pression sur les libéraux anglophones pour obtenir ce respect des droits de la minorité.

Trois ans plus tard cependant, d'Hellencourt acceptait son échec. Non seulement l'aile francophone du parti libéral manitobain était en perte de vitesse ${ }^{13}$, mais encore L'Echo du Mani-

${ }^{11}$ L'Echo de Manitoba (23 juillet 1902), 1.

12 Ibid. Les trois comtés étaient: Saint-Boniface, La Vérendrye et Carillon; et les députés libéraux: S.-A.-D. Bertrand, qui avait d'ailleurs démissionné en novembre 1900 pour se présenter aux élections fédérales où il fut défait par La Rivière; William Lagimodière et Martin Jérôme.

13 Aux élections du 20 juillet 1903, Horace Chevrier ne l'avait emporté que par une voix sur Joseph Bernier dans Saint-Boniface; W. Lagimodière se maintenait de justesse dans La Vérendrye et le conservateur Albert 
toba, qui avait dénoncé la prise de position du clergé en faveur des conservateurs lors des élections de 1903 et qui s'était ainsi gagné bien des inimitiés, allait disparaître. Il ne restait plus à d'Hellencourt qu'à faire ses adieux aux lecteurs:

En somme, ces huit années de lutte ont porté leur fruit.

Désormais le parti libéral français a conquis sa place au soleil du Manitoba.

De proscrit, qu'il était, il est devenu la majorité. Ce résultat est avant tout l'œuvre de L'Echo du Manitoba ${ }^{14}$.

Comme prévu, $L ' E c h o$ ne devait pas survivre longtemps au départ de son rédacteur. Ses adversaires conservateurs assistèrent avec une satisfaction mal contenue sous le couvert d'une benoîte objectivité, à sa rapide agonie:

L'Echo du Manitoba, auquel M. d'Hellencourt faisait ses adieux, le 6 juillet, est de nouveau revenu à ses lecteurs le 13. Le nom de l'ancien directeur n'y apparaît plus. Nous avons aussi remarqué une absence complète d'articles de rédaction. Le journal se borne à des reproductions et à quelques faits divers. Nous donnons ces détails comme manière de renseignements pour nos propres lecteurs. ${ }^{15}$

L'Echo disparut le 20 juillet 1905, pour renaître d'ailleurs sous des livrées différentes. Les libéraux avaient absolument besoin d'un porte-parole lors des campagnes électorales. Le député de Saint-Boniface, Horace Chevrier ${ }^{16}$, finança L'Avenir de l'Ouest; cet hebdomadaire prétendait travailler au "triomphe des idées françaises et catholiques dans l'Ouest canadien" en traitant des thèmes suivants: "éducation, immigration, commerce et agriculture". Une autre feuille éphémère le remplaça: L'OuestCanada ${ }^{17}$ qui fit son apparition en mars 1906, vraisemblablement à l'occasion de la convention libérale du Manitoba tenue à Winnipeg les 27 et 28 mars, puis cessa d'exister en juin de la même année.

Préfontaine gagnait largement dans Carillon. Aux élections du 7 mars 1907, les trois comtés francophones élirent des conservateurs. Il n'y avait que 46 délégués canadiens-français à la convention libérale des 27 et 28 mars 1906 à Winnipeg.

${ }^{14}$ L'Echo du Manitoba (6 juillet 1905), 2.

15 Le Manitoba (19 juillet 1905), 2.

16 Horace Chevrier (1875-1935), né à Ottawa; fils de Noé Chevrier; député provincial de Saint-Boniface, 1903-1907; battu en 1907 par Joseph Bernier; battu en 1910 dans Carillon par Albert Préfontaine; président du "Magasin Bleu" à Winnipeg, 1909-1931; décédé à Saint-Boniface.

${ }^{17} \mathrm{Le}$ titre rappelle, peut-être volontairement, L'Ouest Canadien de Prendergast et Cyr. 
C'est alors qu'une équipe d'immigrants français entra en scène et prit la relève de d'Hellencourt jusqu'en 1914 et même, en plus petit nombre, jusqu'en 1919. Le lyonnais Claudius Juffet fonda Le Nouvelliste, bientôt dirigé par le parisien René Brun et l'angoumois Arthur Boutal. C'était un hebdomadaire qui se voulait "politique et littéraire"; Horace Chevrier fournissait les articles politiques et aussi des fonds. Le Nouvelliste, pour marquer la continuité de la presse libérale, reprenait à son compte la devise de L'Echo, "Tout Droit". Peut-on voir un nouvel hommage rendu à d'Hellencourt, rédacteur politique du Soleil de Québec, dans le changement d'appellation survenu au début d'août 1911 et qui du Nouvelliste fit Le Soleil de l'Ouest? La maladie et la première guerre mondiale décimèrent l'équipe des journalistes.

Et puis, ce fut le retour des libéraux au pouvoir sur la scène provinciale, avec T.C. Norris, à la suite du scandale des édifices du parlement qui fit sombrer le gouvernement Roblin en mai 1915. Les élections qui suivirent (6 août 1915) amenèrent un raz-de-marée libéral. Sans perdre de temps, le gouvernement Norris supprima l'enseignement bilingue au profit de l'anglais dans les écoles du Manitoba, par la fameuse loi Thornton de février-mars 1916. On pouvait croire que le scénario de 1889-1890 se répétait. Les deux députés libéraux canadiens-français se désolidarisèrent ouvertement d'avec le cabinet. La presse libérale d'expression française allait-elle disparaître immédiatement? Le Soleil de l'Ouest s'éteignit le 2 mars, mais La Libre Parole prit aussitôt la relève, avec le même rédacteur, pour promouvoir une "politique indépendante à tendances libérales" et "la lutte pour le français sans acrimonie", ce qui n'allait pas de soi dans le Manitoba de cette époque. Auguste-Henri de Trémaudan, qui avait été rédacteur du Soleil de l'Ouest d'octobre 1915 à la disparition du journal, dirigea La Libre Parole avec la collaboration du professeur Albert Dayen, non sans crises ni démissions, pratiquement jusqu'à la fin, en mars 1919, date à laquelle la presse libérale franco-manitobaine se tut définitivement. ${ }^{18}$

${ }^{18}$ A l'exception de quelques numéros de La Vraie Liberté parus lors des élections de juin 1927 . 


\title{
II - LE RÉDACTEUR : HENRI D'HELLENCOURT
}

\author{
"Cet étranger, qui n'a de nos affaires \\ qu'une conception terne et dénuée de \\ tout intérêt sérieux, nous fait la \\ leçon et nous décerne des certificats \\ d'impuissance et de crétinisme [...]". \\ Le Manitoba (27 août 1902), 2.
}

Curieuse figure et personnalité bouillante que celle de cet officier français, contraint sans doute par une aventure sentimentale à l'exil au Manitoba, et qui n'avait, de toute évidence, aucun goût pour la colonisation agricole, dont il dut subir l'épreuve. Mais la véritable vocation de ce "condottiere du journalisme", comme l'appelle Mgr Langevin ${ }^{19}$, était celle de publiciste et de tribun populaire. Né à Paris le 11 septembre 1862, il fit ses études à Sainte-Croix et au Lycée Henri IV. Il entra à l'école militaire de Saint-Cyr en 1881 et en sortit avec le grade de sous-lieutenant en 1883. Admissible à l'Ecole Supérieure de Guerre en 1890, il donna sa démission pour venir s'établir au Manitoba. Il se fixa à Sainte-Anne-des-Chênes en octobre 1891 et cultiva sans succès la terre jusqu'en $1897^{20}$.

Devenu rédacteur (1898), puis propriétaire (1901) de L'Echo "de" Manitoba, d'Hellencourt qui professait une réelle admiration pour Wilfrid Laurier, entra très vite en communication avec des représentants du gouvernement fédéral. Mgr Langevin, qui n'approuvait ni les idées ni la vie privée du journaliste, lui reprochait d'ailleurs d'avoir "servi le parti libéral afin de gagner sa vie" ${ }^{21}$. Grand partisan du peuplement français de l'Ouest canadien, d'Hellencourt fut chargé par Ottawa d'une mission d'émigration française au Canada et partit pour l'Europe le 10 janvier 1901. Son départ entraîna d'abord une baisse de la qualité des articles, puis une interruption complète de L'Echo du 14 mars au 4 juillet 1901. De retour au Canada, il soumit un rapport intéressant et très favorable à l'immigration française et belge au ministre de l'intérieur, Clifford Sifton, le $11 \mathrm{mai}$ 1901. Dans ce rapport, il posait aussi à mots couverts sa candidature à la succession de Bodard comme agent d'immigration canadien en France.

En décembre 1902, d'Hellencourt touchait au faîte de sa carrière manitobaine. Il était nommé agent consulaire de France

19 AASB (Archives de l'Archevêché de Saint-Boniface), Correspondance Langevin, tome 14 (22 août 1910-22 juillet 1911) : 592-594.

20 L'Echo de Manitoba (24 juillet 1902), 2.

21 AASB, Correspondance Langevin, ibid. 
à Winnipeg. Mais il songeait déjà depuis octobre à quitter le Manitoba pour Montréal. Et c'est à la demande expresse de Laurier qu'il resta à Winnipeg jusqu'en 1905. L'ambitieux journaliste était en effet le partisan inconditionnel de la politique de Wilfrid Laurier et il correspondait régulièrement avec le premier ministre du Canada. Il accepta non sans hésiter la place que lui offrait Laurier, soit le poste de rédacteur en chef du quotidien libéral de Québec, Le Soleil, dont il assuma la direction politique de 1906 à 1920 . Il aurait préféré le milieu social montréalais pour des raisons familiales.

Pendant la première guerre mondiale, il rédigea, sur le déroulement des hostilités, des chroniques quotidiennes qui lui valurent de grands éloges. Après un séjour en France, il revint au Canada et devint rédacteur à La Presse de Montréal jusqu'en 1928, date à laquelle il prit sa retraite en France, à Paris et à Thouars (Deux-Sèvres). Il partagea ses dernières années entre la capitale et la tranquille cité du Poitou jusqu'à sa mort, survenue au moment où s'effondrait "une certaine idée de la France", le 8 mai $1940 .{ }^{2}$

\section{III - LES PRINCIPAUX THEMMES DU JOURNAL}

Il n'est pas question, dans le cadre d'un simple survol, de faire une analyse du contenu de L'Echo du Manitoba ${ }^{23}$. Il suffit de signaler quelques thèmes qui reviennent familièrement sous la plume du rédacteur et qu'il reprend de façon synthétique dans ses adieux aux lecteurs.

\section{A. Soutien inconditionnel à Wilfrid Laurier}

Le principal thème politique est sans aucun doute le soutien inconditionnel accordé à Wilfrid Laurier et au parti libéral. Comme l'écrit d'Hellencourt au moment de quitter le Manitoba:

${ }^{22}$ La Liberté (5 juin 1940), 10.

23 Nous n'évoquerons pas ici les problèmes de politique extérieure, comme la guerre des Boers, la guerre russo-japonaise qui passionnait le saint-cyrien d'Hellencourt au point que Le Manitoba le surnommait "le général en chef des armées russes au Canada", ou encore la question des relations franco-canadiennes et l'image de la' France d'après L'Echo (l'hebdomadaire acceptait l'héritage révolutionnaire et la République, il refusait de dissocier la France officielle de la France catholique: "Il n' a qu'une seule France", titrait l'Echo lors d'une polémique sur le sujet en juillet 1903). 
J'ai foi, j'ai confiance dans le parti libéral canadien. Cette foi, cette confiance ont leur source dans l'étude des faits, dans la connaissance du chef qui dirige ce parti.

Mais jamais je n'ai cessé d'exercer librement mon jugement, même à l'égard de notre parti.

Quand M. Tarte, profitant de certaines circonstances trop aisément escomptées par son ambition, entreprit la campagne qui se termina par sa démission, je fus le premier et le seul des journalistes canadiens-français à le dénoncer et à le combattre.

Quand M. Sifton provoqua, par sa démission, la funeste crise que l'on sait nous n'hésitâmes pas à parler franc et net. ${ }^{24}$

Ce dévouement au parti libéral et à son prestigieux leader entraînait logiquement une hostilité virulente contre les conservateurs franco-manitobains et précisément contre les rédacteurs du journal Le Manitoba.

\section{B. Refus du dirigisme clérical en politique}

Mgr Langevin et le clergé de la province favorisaient le parti conservateur du Manitoba au détriment des libéraux, malgré le compromis Laurier-Greenway et l'encyclique "Affari Vos" qui conseillait de l'accepter comme un moindre mal. D'Hellencourt refusa donc systématiquement à la hiérarchie ecclésiastique, surtout depuis les élections provinciales de 1903, le droit de diriger les consciences dans le domaine de la politique. Le raisonnement d'un Français, installé depuis 1899 au Manitoba, et qui conseillait, au cours d'une promenade après les vêpres, à son ami Charles Regnard, catholique et Français comme lui, de se ranger "derrière le défenseur de la vérité en ce diocèse [...] même pour la politique", devait faire bondir d'Hellencourt. Le Français en question affirmait, qu'en politique comme en matière de foi, la société des fidèles devait "une obéissance prompte et entière au Pape, aux évêques et aux prêtres". Regnard ne sut que répondre, car le fidèle, pensait-il, doit entière soumission à son évêque et appartient à l'Eglise avant d'être à sa patrie et à sa famille; il est avant tout un chrétien, comme électeur, comme père de famille et comme travailleur; et dans tous ces

24 L'Echo du Manitoba (6 juillet 1905), 2. Les Archives publiques du Canada possèdent la correspondance Laurier-d'Hellencourt de 1900 à 1919. Pendant le séjour manitobain du journaliste, 70 lettres furent échangées entre les deux hommes et 5 entre Laurier et Mme d'Hellencourt. 
domaines, le chrétien doit suivre les directives de la hiérarchie ecclésiastique..$^{25}$

Face à cette confusion du temporel et du spirituel, le rédacteur de L'Echo ne tarda point à éclairer de ses lumières l'esprit obscurci de Regnard, "dans l'intérêt même de la religion". Il le faisait d'autant plus volontiers que la thèse soutenue dans $L e$ Manitoba jouissait "d'une faveur particulière dans le diocèse" et que certains ecclésiastiques ne manquaient "aucune occasion de s'en faire les apôtres". Certes, concédait le rédacteur, c'est une bonne chose de se renseigner, avant de voter, auprès de son curé ou de son évêque, qui possèdent une influence légitime en tant que chefs spirituels, mais nul fidèle n'est tenu à "l'obligation forcée d'obéissance à ces mêmes chefs spirituels en matière politique". Pourquoi? Parce que la société ne confère le droit de vote qu'aux êtres intelligents et libres, et non aux idiots, aux indignes, à tous ceux qui ne peuvent exercer leur libre arbitre.

Piqué au vif par cet article, où transparaissait la condescendance du publiciste bien informé, Charles Regnard répondit avec un beau respect de la logique formelle. Il revendiquait, au nom du libre arbitre, son droit d'abdiquer sa liberté de conscience politique entre les mains de l'Archevêque. Et il concluait en disant qu'il préférait suivre les recommandations de son évêque en politique plutôt que les conseils du "premier charlatan venu".

Quant à d'Hellencourt, il réaffirma clairement sa position envers le clergé catholique dans son dernier éditorial:

Je crois fermement, et je l'ai déjà proclamé, que l'influence du prêtre doit être le facteur dominant dans le développement de la race française au Canada.

Mais pour être durable, et bienfaisante, cette influence dans les choses du monde doit, non point être imposée, mais s'imposer d'elle-même.

Il faut, il est nécessaire que le prêtre ne soit pas seulement l'aviseur spirituel, mais aussi le guide naturel du peuple et ce rôle il ne peut le remplir qu'en planant au-dessus des partis et des intérêts. ${ }^{26}$

\section{Propagande pour l'immigration francophone}

L'Echo du Manitoba fit également campagne en faveur de l'immigration française et belge, au Canada en général et au

${ }^{25}$ Le Manitoba (8 juillet 1903), 1.

${ }^{26}$ L'Echo du Manitoba (6 juillet 1905), 2. 
Manitoba en particulier. D'Hellencourt avait même accepté, comme nous l'avons signalé, pour le gouvernement fédéral une mission d'enquête sur les possibilités offertes par la France et la Belgique et il rêvait de devenir agent d'immigration canadien en France. Ainsi L'Echo du 8 août 1901 titrait sur six colonnes: "L'Ouest c'est l'avenir".

Le rédacteur avait d'ailleurs composé un numéro spécial de 16 pages, en août 1900, sur la colonisation française au Manitoba et les possibilités qui s'offraient à l'immigration. On y trouve des monographies de plusieurs paroisses, en particulier SainteRose-du-Lac, Saint-Claude, Notre-Dame-de-Lourdes, Saint-Laurent, Fannystelle et Laurier. Mgr Langevin avait accepté de collaborer à ce numéro et il y lançait un appel à la colonie québécoise. Immédiatement après venait l'article de d'Hellencourt sur l'immigration française. Le paysan français, dur à la peine et attaché au sol, notait le rédacteur, est le colonisateur idéal mais il a un défaut majeur, capital à vrai dire: il n'émigre pas. Il faut donc faire appel à l'ouvrier agricole, qui pourrait devenir propriétaire au Manitoba. Par contre, il faut exclure de la colonisation agraire "les commis de magasins, les petits commerçants, tous les jeunes gens de profession libérale", et aussi les "fils de famille, que les parents envoient en exil, au Manitoba, pour les punir de leurs fredaines", car "le Manitoba n'est pas une colonie de déportation". D'Hellencourt ne limitait pas sa vision au milieu rural, il tournait aussi ses regards vers le monde des affaires. Les Français détenteurs de capitaux peuvent espérer de gros intérêts, de 6 à $10 \%$, s'ils placent leur argent au Manitoba. Les maisons de commerce françaises pourraient ouvrir des succursales dans la province à condition "d'envoyer sur place un agent sérieux parlant l'anglais", car:

Le luxe à Winnipeg est des plus développés, surtout le luxe féminin et les articles de mode et de confections, les linges, rubans, dentelles, ameublement, qui sont la gloire de l'industrie française, auraient ici un débouché lucratif. 27

Dans son rapport du 11 mai 1901, consacré uniquement à la colonisation agricole, d'Hellencourt développait cinq points principaux:

$1^{\circ}$ L'immigration française est désirable mais difficile. Le paysan français, ouvrier agricole ou fermier, constitue "un excellent colon, travailleur, économe, persévérant, qui, de plus, a cette

${ }^{27}$ L'Echo de Manitoba (numéro spécial d'août 1900). Maurice Constantin-Weyer évoque cette atmosphère des années 1905-1914 à Winnipeg dans le roman Un homme se penche sur son passé (Paris, Rieder, 1929). 
qualité précieuse de s'attacher au sol qu'il cultive". Les colonies de Notre-Dame-de-Lourdes, Saint-Claude, Grande-Clairière et Sainte-Rose-du-Lac, au Manitoba, en sont des exemples. Cependant le paysan français s'expatrie peu et il se méfie de la propagande des agents d'immigration.

$2^{\circ}$ Il faut donc trouver un bon agent d'immigration canadien en France pour remplacer Auguste Bodard. L'agent devra se renseigner sur les lieux d'origine des immigrants français, "comme j'ai eu l'occasion de le faire moi-même", souligne d'Hellencourt. Chaque année, des colons ayant très bien réussi seraient envoyés en France par le gouvernement canadien: "ils travailleraient de concert avec l'agent d'immigration qui les emmènerait en tournée à travers la campagne, comme exemples vivants, afin de gagner la confiance des paysans français". L'expérience a été couronnée de succès en Belgique. Ce moyen serait également efficace en France.

$3^{\circ}$ Trois nouvelles conditions faciliteront l'émigration française. Tout d'abord une ligne de navigation directe vient d'être établie entre la France et le Canada. Ensuite, on discerne une certaine inquiétude chez la population rurale française. Enfin il faut insister auprès des paysans français sur la facilité avec laquelle on peut devenir propriétaire terrien au Canada: “c'est à notre avis le point le plus important à développer si l'on veut faciliter l'immigration française au Canada."

$4^{\circ}$ Le taux de natalité nettement excédentaire que certaines régions françaises ont conservé jouera en faveur de l'émigration. Ce sont ces régions que l'agent d'immigration doit parcourir, en particulier le pays basque, les départements du Nord, la Bretagne et la Loire-Inférieure, l'Auvergne, la Franche-Comté, la Savoie, le Limousin, les Vosges et les Ardennes.

$5^{\circ}$ Trois moyens sont à utiliser et deux erreurs sont à éviter pour favoriser l'immigration. Il faut d'abord adapter les brochures de propagande aux paysans français et non traduire celles destinées aux Irlandais, aux Anglais ou aux Canadiens des EtatsUnis; ces brochures seront brèves, de petit format, de forme concise, et contiendront surtout des témoignages de colons français déjà établis au Canada. Il faut ensuite faire beaucoup de publicité dans la presse, pas dans la presse provinciale, mais dans les journaux à bon marché et à gros tirage, comme Le Petit Journal ou Le Petit Parisien; une carte du Canada, avec l'annonce des homesteads offerts aux colons, devrait paraître trois ou quatre fois par semaine dans les journaux. L'agent d'immigration devra enfin donner des conférences publiques, avec ou 
sans cartes, devant les bourgeois des villes, car le paysan français consulte les notables de l'endroit avant d'émigrer. Deux erreurs sont à éviter: pour attirer les colons dans une région du Canada, on se laisse aller à décrier les régions voisines: à ce jeu, toutes les provinces acquièrent rapidement la réputation d'être inhospitalières; certains agents d'immigration favorisent une compagnie de navigation aux dépens des autres: cette pratique entraîne la corruption de l'agent au détriment de l'immigration.

Grâce à un travail mené suivant ces recommandations, conclut d'Hellencourt, on pourrait espérer voir débarquer un contingent de deux mille colons français au Canada. Cependant en 1903, c'est Paul Wiallard, et non d'Hellencourt, qui fut nommé par le Gouvernement canadien, agent d'immigration et souscommissaire du Canada à Paris.

D'Hellencourt dénonçait aussi les obstacles dressés par la France officielle et les Anglo-Canadiens contre cette immigration. A Winnipeg même, l'agent consulaire de France, Frédéric Gautier, fidèle en cela à la politique préconisée par le consul général Kleczkowski, ${ }^{28}$ menait une campagne discrète contre l'émigration française ou du moins ne cherchait pas du tout à l'encourager. Il allait jusqu'à écrire, dans le numéro spécial de $L ' E c h o$ consacré à la colonisation :

Je dis tout d'abord, en ce qui touche l'émigration française que je suis loin de préconiser le départ des Français de France pour l'étranger, ils n'y trouvent, le plus souvent, que la déception et la misère: que je préfère les voir se diriger vers nos colonies françaises et aider à y fonder des établissements prospères pour le commerce de la mère-patrie, et par là assurer la prospérité et la grandeur de la métropole.

\section{CONCLUSION}

La presse libérale franco-manitobaine est née, si l'on en croit le discours qu'elle tient sur elle-même, de la volonté d'un groupe de Manitobains désireux d'exposer leur point de vue aux anglophones de la province. Il leur fallait, disaient-ils, non pas opposer une race minoritaire à une race majoritaire, mais s'intégrer dans le jeu des partis politiques pour défendre leur cause linguistique et scolaire auprès de ceux-ci. Un groupe francophone trop exclusivement lié au parti conservateur leur semblait

28 Pierre Savard, Le Consulat général de France à Québec et à Montréal de 1859 à 1914 (Québec, PUL, 1970), 87-93. 
dangereusement menacé en cas de prise de pouvoir par les libéraux. Les hebdomadaires libéraux successifs se donnèrent pour but de soutenir cette aile française de la famille libérale, de la rendre influente au sein de la minorité et crédible électoralement auprès des libéraux anglais. Ce but a-t-il été atteint? ${ }^{29}$ Ils avaient

29 Pour déterminer l'influence de ces hebdomadaires auprès des francophones, il faudrait faire une étude systématique de la correspondance des curés de paroisse, ou d'autres personnes, avec l'Archevêque de SaintBoniface. Par exemple, le 29 décembre 1899, l'abbé Jean Gaire, curé de Grande-Clairière, écrivait à $\mathrm{Mgr}$ Langevin et cherchait à comprendre pourquoi les trois comtés français (Saint-Boniface, La Vérendrye et Carillon) avaient élu des libéraux: "Nous ne pouvons permettre qu'on abuse du vote de ceux de la Rivière-Rouge. L'Echo de Manitoba les a trompés en bonne partie, le reste c'était des brebis galeuses." Après les élections provinciales du 20 juillet 1903 , qui avaient également porté au pouvoir des libéraux dans trois circonscriptions françaises (Saint-Boniface, La Vérendrye et Assiniboia), A. Audet (1840-1918, colonel, organisateur du parti conservateur; voir RHAF (septembre 1947): 271-277) écrivait d'Ottawa à Mgr Langevin le 22 juillet: "Il y a un vice quelque part, un élément délétère qu'il faudrait faire disparaître. [...] Est-ce l'effet dissolvant de L'Echo du Manitoba ?" Il faudrait étudier aussi l'implantation des comités libéraux locaux et leur action.

Quant aux libéraux anglophones, ils semblent n'avoir pas pris très au sérieux la presse partisane de langue française. D'Hellencourt se plaignait en effet, dans une lettre du 10 décembre 1900 à J. Obed Smith (fonctionnaire travaillant au bureau d'immigration à Winnipeg) d'avoir $\mathrm{vu}$ ses services ignorés lors des élections fédérales par les responsables locaux de l'organisation du parti:

For Selkirk, Mr McCreary took in hand all business and never asked from me [...]

In McDonald, $\mathrm{Mr}$ Brown replied to me 4th october that he did not require my services, very few French voters being located in his constituency.

The 6th I answered that over 250 French voters are located in St Rose (sic), Laurier, McCreary, St Mare (sic) and Rathwell.

I did not receive any word [...]

Neverthless, without order, I have send from the first day, literature and copies of "L'Echo" in St Rose, Laurier, Rathwell etc. etc.

What I can do, when the local orga $[n]$ izer refuse my services. Now for Lisagr (sic, pour Lisgar).

Day after day I send to each member of committee for distribution, lot of pamphlets and documents, and each week, lot of "L'Echo" [APC, Fonds Sifton, MG 27 II D15, 81: 61738-61739]. 
aussi comme but inavoué mais non négligeable de permettre aux ambitieux opposés aux gens en place, en l'occurrence les conservateurs, de tenter leur chance sur la scène politique locale.

Il ne faut pas négliger non plus l'appel de la race:

In 1885, however, the Conservative Government in Ottawa let Louis Riel be hanged and, two years later, Wilfrid Laurier became leader of the Liberal Party. Both events increased the appeal of the Liberal party among French Canadians. ${ }^{30}$

En effet, la durée de cette presse libérale coïncide à peu près exactement avec le passage de Laurier à la direction du Parti libéral. Vers le 20 mars 1919, quelques semaines après la mort de Laurier, survenue le 17 février, le dernier hebdomadaire libéral de langue française du Manitoba, La Libre Parole, disparaissait à son tour: rencontre symbolique. Peut-on dire cependant qu'il n'y a eu de presse libérale franco-manitobaine que grâce à Laurier? De l'existence de cette presse, son leadership fut en tout cas la condition nécessaire. Car sur le plan provincial, son histoire est encadrée par les attaques systématiques des libéraux anglais contre la minorité française, avec les agressions caractérisées de 1889-1890 et de 1916. La première offensive entraîna la disparition totale de l'hebdomadaire libéral de langue française de 1889 à 1898, et l'assaut de 1916 son effacement avec sursis de trois ans, sursis dû sans doute à Laurier, dont La Libre Parole fait l'éloge tout en prenant ses distances vis-à-vis des libéraux provinciaux. Seules l'arrivée de Laurier au pouvoir en 1896 et sa politique de limitation des dégâts linguistiques et scolaires au Manitoba rendirent crédibles une section française du parti libéral provincial et la presse qui en était l'organe. Ce qui explique ce paradoxe: la belle époque de la presse libérale de langue française fleurit sous le règne des conservateurs provinciaux (1899-1915) assez peu portés à attaquer la minorité francophone, mais solidaires au fond des basses œuvres linguistiques exécutées par les libéraux.

Ce succès relatif est dû aussi, semble-t-il, à la présence de nombreux Français récemment immigrés qui fournirent des rédacteurs, des correspondants, des lecteurs et aussi des opposants farouches à la presse libérale. ${ }^{31}$ Les hebdomadaires libéraux qui ont réussi à tenir pendant plusieurs années étaient rédigés par

30 Roger Turenne, The Minority and the Ballot Box, 14.

$31 \mathrm{Au}$ recensement de 1911, il y a au Manitoba 30,944 francophones, dont 3,146 Français nés en France, soit 10\% des Franco-Manitobains. 
ces cousins de France qu'étaient Henri d'Hellencourt, Claudius Juffet, René Brun, Arthur Boutal, Auguste-Henri de Trémaudan et Albert Dayen ${ }^{32}$. Plus que leur qualité d'étrangers ${ }^{33}$, c'est leur option politique qui leur valut une méfiance certaine de la part du clergé et de la fraction conservatrice de la population, car d'autres Français comme Jules Grymonpré et François Deniset participèrent à la rédaction du Manitoba où ils furent accueillis à bras ouverts ${ }^{34}$. Après le départ ou la mort de ces rédacteurs, aucun Manitobain ne prit leur relève. Les énergies locales se tournèrent plutôt du côté du nouvel hebdomadaire La Liberté, fondé à la demande de Mgr Langevin pour réaliser l'union de tous les Canadiens français sur le terrain national et religieux, en dehors des partis politiques, comme Le Métis à ses débuts et avec la même devise: "Dieu et mon droit". Après la disparition du Manitoba, le 29 juillet 1925, la boucle était bouclée: c'était le retour au monopole d'un journal, qui s'identifiait à la défense de la minorité et non plus au soutien partisan d'une famille politique. L'expression des opinions y perdit en variété et en combativité. En journalisme comme en politique, le meil-

32 En 1923, un autre Français immigré, Donatien Frémont, qui avait été rédacteur du Patriote de l'Ouest, en Saskatchewan, de 1916 à 1923, allait prendre la direction de La Liberté et la conserver pendant 18 ans.

33 Certains Français, même ecclésiastiques, se plaignent de la francophobie de la classe dirigeante canadienne-française. Ainsi, le 18 juillet 1900, un prêtre français, l'abbé J.-M. Jolys, curé de Saint-Pierre au Manitoba, écrivait à un autre prêtre français, dom Paul Benoît, curé de NotreDame-de-Lourdes, à propos de sa brochure sur L'Anglomanie au Canada:

Je connais depuis longtemps jusqu'où peut aller l'anglomanie et surtout la Francophobie même chez certains de ceux qui se disent les amis de la France. [...]

Mon bien cher père, j'ai eu, dans mes nombreuses relations avec les Canadiens français l'extrême douleur de constater chez plusieurs principalement dans la classe dirigeante une Anglomanie d'une espèce particulière qui nous fait rencontrer dans le même être un certain amour de la France uni à une certaine rancune envers la terre des ayeux s'amalgamant à une certaine admiration des coutumes anglaises, de la grandeur anglaise et [ici est le venin] de la liberté anglaise. [AASB, Fonds Benoît (Guéret)].

34 AASB, Fonds Langevin, Réunion des évêques (1899-1909). Réunion épiscopale tenue à l'Archevêché de Saint-Boniface sous la présidence de Mgr l'Archevêque les 9, 10, 11, 12 et 13 septembre 1903: “... 15) Mgr l'Archevêque demande que l'on s'efforce, de toute manière possible, d'empêcher la diffusion du journal "L'Echo du Manitoba", qui est rédigé dans un très mauvais esprit." 
leur facteur de dynamisme n'est-il pas la présence d'un adversaire vigilant?*

* Je tiens ici à exprimer toute ma gratitude à Mgr Baudoux, qui met libéralement les archives de l'Archevêché de Saint-Boniface à la disposition des chercheurs. Merci également à Lionel Dorge et à Robert Painchaud: leur incomparable connaissance des AASB et leur sens du travail en équipe m'ont permis de prendre connaissance de pièces qui constituent le principal intérêt de cette communication. 